\title{
Thyroid Function Abnormalities during Amiodarone Therapy for Persistent Atrial Fibrillation
}

\author{
Elizabeth L. Batcher, MD, ${ }^{a}$ X. Charlene Tang, MD, PhD, ${ }^{b}$ Bramah N. Singh, MD, DSc, ${ }^{a}$ Steven N. Singh, MD, ${ }^{c}$ \\ Domenic J. Reda, PhD, ${ }^{b}$ Jerome M. Hershman, MD ${ }^{a}$ for the SAFE-T Investigators \\ ${ }^{a}$ West Los Angeles Veterans Affairs Medical Center, Los Angeles, Calif, ${ }^{b}$ Edward Hines, Jr. Veterans Affairs Hospital, Hines, Ill, and \\ ${ }^{c}$ Department of Veterans Affairs Medical Center, Washington, DC.
}

\begin{abstract}
BACKGROUND: Many patients receiving amiodarone therapy are male. The long-term risk for amiodarone-induced thyroid dysfunction in these patients has not been systematically and prospectively investigated. The purpose of this study was to determine the extent of amiodarone-induced thyroid dysfunction in a large male cohort.

METHODS: This is a substudy of a prospective randomized controlled trial (SAFE-Trial) in which amiodarone, sotalol, and placebo for persistent atrial fibrillation were evaluated. For the purpose of this substudy, sotalol and placebo groups were combined into a control group. Serial thyroid function tests were performed over 1-4.5 years. Of the 665 patients enrolled in the SAFE-Trial, 612 patients were included in this sub-study.

RESULTS: Subclinical hypothyroidism, thyroid-stimulating hormone (TSH) level 4.5-10 mU/L, was seen among $25.8 \%$ of the amiodarone-treated patients and only $6.6 \%$ of controls $(P<.0001)$. Overt hypothyroidism, TSH level $>10 \mathrm{mU} / \mathrm{L}$, was seen among $5.0 \%$ of the amiodarone-treated patients, and only $0.3 \%$ of controls $(P<.001)$. By 6 months, $93.8 \%$ of the patients who developed TSH elevations above 10 $\mathrm{mU} / \mathrm{L}$ on amiodarone had been detected. There was a trend toward a greater proportion of hyperthyroidism, defined as a TSH $<0.35 \mathrm{mU} / \mathrm{L}$, in the amiodarone group compared with the control group (5.3\% vs $2.4 \%$, $P=.07)$.

CONCLUSIONS: Hypothyroidism developed in $30.8 \%$ of older males treated with amiodarone and in only $6.9 \%$ of the controls. Hypothyroidism presented at an early stage of therapy. Hyperthyroidism occurred in $5.3 \%$ of amiodarone treated patients, and was a subclinical entity in all but 1 case. (C) 2007 Elsevier Inc. All rights reserved.
\end{abstract}

KEYWORDS: Amiodarone; Hypothyroidism; Sotalol; Thyroid

Although the unique pharmacologic properties of amiodarone and its varied clinical uses have been known for several decades, its unparalleled effectiveness in maintaining sinus rhythm has emerged only in recent years. The drug's major shortcoming has been its propensity to induce disorders of thyroid function. It causes various forms of thyroid

\footnotetext{
Supported by the Cooperative Studies Program of the Department of Veterans Affairs office of Research and Development.

Requests for reprints should be addressed to Elizabeth Batcher, MD, Department of Medicine, UCLA-Olive View Medical Center, 14445 Olive View Drive, Sylmar, CA, 91342.

E-mail: batcherb@gmail.com
}

dysfunction ranging from elevated serum thyroxine (T4) levels and low triiodothyronine (T3) levels in euthyroid patients ${ }^{1}$ to overt hypothyroidism or hyperthyroidism. The primary cause of the thyroid dysfunction is the large iodine load that can cause either hypothyroidism or hyperthyroidism. ${ }^{2,3}$ Hyperthyroidism can also be caused by a thyroiditis that is iodine-independent, and known as type 2 hyperthyroidism. ${ }^{2,3}$

Studies examining the prevalence of amiodarone-induced thyroid dysfunction vary in regard to geographic location and iodine intake, ratio of women to men, and length of follow-up. Most studies have shown a trend toward more frequent hypothyroidism in iodine-sufficient areas, such as the 
United States, and more frequent thyrotoxicosis in iodinedeficient areas, such as Italy. ${ }^{4,5}$ In general, amiodarone-induced thyroid dysfunction is more common in patients with an underlying thyroid disorder. Rates of thyroid dysfunction have been reported to be $14 \%-18 \%$, with the highest prevalence among women and those with antithyroid antibodies. ${ }^{2-4,6}$

The data reported in this article deal with thyroid dysfunction in a large cohort of male patients treated with amiodarone and compared with a control group stemming from a substudy of the Sotalol Amiodarone Atrial Fibrillation Efficacy Trial (SAFE-T). ${ }^{7}$ The objective of the substudy was to determine the incidence of hypothyroidism, defined as thyroid-stimulating hormone $(\mathrm{TSH})>4.5 \mathrm{mU} / \mathrm{L}$, or hyperthyroidism, defined as TSH $<0.35$ $\mathrm{mU} / \mathrm{L}$, induced by amiodarone and compared with sotalol and placebo as the control.

\section{METHODS}

\section{CLINICAL SIGNIFICANCE}

- Nearly one third of older men treated with amiodarone for persistent atrial fibrillation developed some degree of hypothyroidism, compared with $6.9 \%$ of those treated with sotalol or placebo.

- Amiodarone treatment also was associated with a slight, nonsignificant increase in hyperthyroidism ( $5.3 \%$ vs $2.4 \%$ ).

- During amiodarone therapy, all patients should be carefully monitored for both hypothyroidism and hyperthyroidism.

- Amiodarone-induced overt hypothyroidism generally warrants levothyroxine treatment.

\section{Study Design}

The details of the study (SAFE-T) design from which the data for the current report are derived have been reported previously. ${ }^{7}$ In brief, patients with persistent atrial fibrillation (AF) were randomly assigned to amiodarone, sotalol, or placebo after optimal anticoagulation (international normalized ratio ranging from 2 to 3 ). After 4 weeks of therapy, those not in sinus rhythm (SR) had their AF electrically cardioverted. Once SR was achieved, anticoagulation was continued for at least 8 weeks. Recurrence of AF was documented by weekly transtelephonic monitoring. All patients were followed for at least 1 year regardless of their rhythm status. Patients remaining in SR were maintained on their originally assigned treatment. Those who remained in, or reverted to, AF after second cardioversion were placed on open-label medications with continued follow-up to 1 year. Thyroid function was examined at baseline, 3 months, 6 months, and at every 6 months thereafter by measurement of serum thyrotropin (TSH) concentration. Free $\mathrm{T} 4$ and total T3 levels were not consistently obtained.

\section{Statistical Analysis}

Incidence rates of hypothyroidism and hyperthyroidism between groups were compared using chi-squared test, or Fisher's exact test as appropriate. Logistic regression was used to calculate odds ratios. An alpha value of .05 or less was considered to indicate statistical significance. All statistical tests reported were 2-sided. All analyses were performed using SAS version 8.0 (SAS Institute Inc., Cary, NC). disease, smoking, or baseline TSH level between the groups. Rates of baseline levothyroxine therapy were similar between the control and amiodarone-treated groups, $1.4 \%$ versus $2.8 \%$, respectively $(P=.20)$. The pairwise comparisons showed that there were no statistical differences in baseline TSH between original treatment groups.

Sixty-six of 365 patients in the control group received open-label amiodarone for at least 1 month during follow-up and were excluded from the outcome analysis in

Table 1 Baseline Patient Characteristics

\begin{tabular}{|c|c|c|c|}
\hline Characteristics & $\begin{array}{l}\text { Amiodarone } \\
\text { Group } \\
(n=247)\end{array}$ & $\begin{array}{l}\text { Control } \\
\text { Group } \\
(n=365)\end{array}$ & $\begin{array}{l}P \\
\text { Value }\end{array}$ \\
\hline Age & $67.1 \pm 9.4$ & $66.9 \pm 9.2$ & .84 \\
\hline \multicolumn{4}{|l|}{ Sex } \\
\hline Male (\%) & $246(99.6)$ & $364(98.9)$ & .65 \\
\hline Body mass index $\left(\mathrm{kg} / \mathrm{m}^{2}\right)$ & $31.3 \pm 5.8$ & $31.5 \pm 5.9$ & .67 \\
\hline Current smokers $(\%)$ & $4(1.6)$ & $3(0.8)$ & .45 \\
\hline Diabetes (\%) & $62(25.2)$ & $90(24.7)$ & .88 \\
\hline Ischemic HD (\%) & $64(25.9)$ & $88(24.1)$ & .61 \\
\hline \multicolumn{4}{|l|}{$\begin{array}{l}\text { Baseline TSH } \\
\qquad(\mathrm{mU} / \mathrm{L})(\%)\end{array}$} \\
\hline Low: $<0.35$ & $5(2.1)$ & $3(0.9)$ & .18 \\
\hline Normal: 0.35 to 4.5 & $231(95.0)$ & $334(93.8)$ & \\
\hline High: $>4.5$ & $7(2.9)$ & $19(5.3)$ & \\
\hline Use of levothyroxine (\%) & $7(2.8)$ & $5(1.4)$ & .24 \\
\hline
\end{tabular}


Table 2 Incidence of Hyperthyroidism and Hypothyroidism During Follow-up*

\begin{tabular}{|c|c|c|c|}
\hline TSH Level (mU/L) & $\begin{array}{l}\text { Amiodarone } \\
\text { Group } \\
n=247 \\
\%(n)\end{array}$ & $\begin{array}{l}\text { Control } \\
\text { Group } \\
n=299 \\
\%(n)\end{array}$ & $\begin{array}{l}P \\
\text { Value }\end{array}$ \\
\hline \multicolumn{4}{|l|}{$\begin{array}{l}\text { Hyperthyroidism } \\
\qquad(\text { TSH }<0.35)\end{array}$} \\
\hline Yes & $5.3(13) \dagger$ & $2.4(7) \dagger$ & .07 \\
\hline No & $94.7(232)$ & $97.6(291)$ & \\
\hline \multicolumn{4}{|c|}{$\begin{array}{l}\text { Subclinical hypothyroidism } \\
\qquad(\mathrm{TSH}>4.5-10)\end{array}$} \\
\hline Yes & $25.8(62) \ddagger$ & $6.6(19) \ddagger$ & \\
\hline No & $74.2(178)$ & $93.4(271)$ & $<.0001$ \\
\hline \multicolumn{4}{|c|}{$\begin{array}{l}\text { Overt hypothyroidism } \\
\qquad(\text { TSH }>10)\end{array}$} \\
\hline Yes & $5.0(12) \ddagger$ & $0.3(1) \ddagger$ & \\
\hline No & 95.0 & $99.7(289)$ & $<.001$ \\
\hline \multicolumn{4}{|c|}{$\begin{array}{l}\text { TSH }=\text { thyroid-stimulating hormone. } \\
\text { *Excluding } 66 \text { patients with open-label amiodarone from the con- } \\
\text { trol group. } \\
\text { †Four patients who had low TSH at both baseline and follow-up } \\
\text { were excluded from analysis. } \\
\quad \text { TTwenty patients who had elevated TSH at both baseline and } \\
\text { follow-up were excluded from analysis. }\end{array}$} \\
\hline
\end{tabular}

Table 2, Figure 1, and Figure 2. Those with open-label amiodarone tended to be younger (64.8 \pm 9.3 years) and had a higher body mass index $\left(33.9 \pm 6.1 \mathrm{~kg} / \mathrm{m}^{2}\right)$ compared with the remaining 299 patients in the control group $(P=.04$, $P=.002$, respectively). There were no significant differences in other demographic and clinical characteristics between the patients with open-label amiodarone and the remaining patients in the control group.

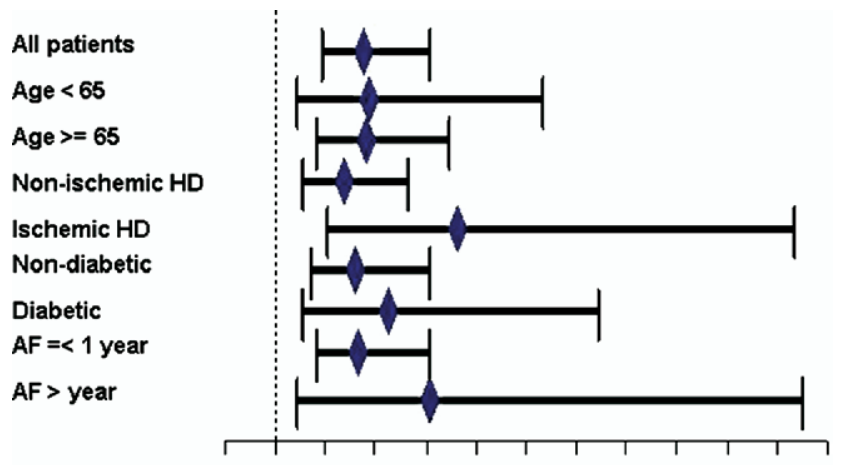

$\begin{array}{lllllllllllll}-1 & 1 & 3 & 5 & 7 & 9 & 11 & 13 & 45 & 17 & 19 & 21 & 2\end{array}$

Figure 1 Figure reflects an updated analysis in which 66 patients with open-label amiodarone were excluded from the control group. Odds ratio (OR) and 95\% confidence limits of hypothyroidism $(\mathrm{TSH}>4.5 \mathrm{mU} / \mathrm{L}$ ) in the amiodarone group compared with the control group. Overall, patients on amiodarone had a higher odds of hypothyroidism than those who were in the control group (odds ratio 4.5, 95\% confidence limit 2.8-7.2). Odds of hypothyroidism was significantly higher for the amiodarone group than for the control group in each predefined stratum. Twenty patients who had elevated TSH at both baseline and follow-up were excluded from analysis.

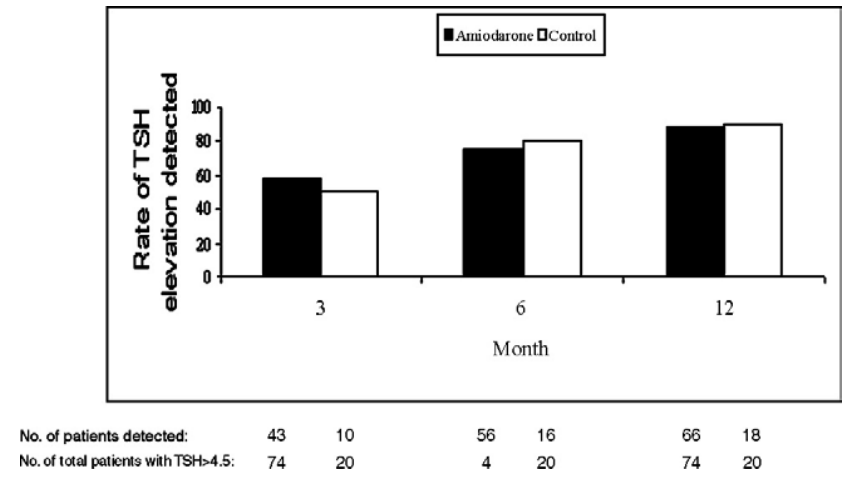

Figure 2 Figure reflects an updated analysis in which 66 patients with open-label amiodarone were excluded from the control group. Rate of hypothyroidism (TSH $>4.5 \mathrm{mU} / \mathrm{L}$ ) for the amiodarone and control groups at 3,6,12, and $>12$ months follow-up.

\section{Incidence of Hypothyroidism and Hyperthyroidism}

Table 2 presents the incidence of hypothyroidism and hyperthyroidism for patients in the amiodarone group and the control group. An elevation in TSH was classified into 2 levels: subclinical hypothyroidism, TSH $>4.5-10 \mathrm{mU} / \mathrm{L}$; and overt hypothyroidism, TSH $>10 \mathrm{mU} / \mathrm{L}$. There was a higher incidence of subclinical hypothyroidism $(25.8 \%)$ in the amiodarone group compared with the control group $(6.6 \%)(P<.0001)$. An elevation of TSH $>10 \mathrm{mU} / \mathrm{L}$ was seen among $5.0 \%$ of the amiodarone-treated patients and only $0.3 \%$ of controls $(P<.001)$, indicating that the patients on amiodarone were more likely to develop overt hypothyroidism than those on placebo or sotalol. As hypothesized, there was no significant difference in the incidence of hypothyroidism between the sotalol- and placebo-treated patients (data not shown).

Figure 1 shows the odds ratio of all degrees of hypothyroidism (TSH $>4.5$ ) for all amiodarone-treated patients and each subgroup of these patients in terms of age, ischemic heart disease, diabetes, and duration of AF before the treatment compared with the control group in which the patients remained on sotalol or placebo. Overall, patients on amiodarone had a higher likelihood to develop hypothyroidism compared with those in the control group (odds ratio 4.5, 95\% confidence limit 2.8-7.2). The likelihood of developing hypothyroidism in patients on amiodarone was significantly higher than that in patients who were in the control group in all predefined strata.

There was a trend toward a higher incidence of hyperthyroidism, defined as a TSH $<0.35 \mathrm{mU} / \mathrm{L}$, in patients on amiodarone compared with those in the control group (5.3\% vs $2.4 \%, P=.07$ ) (Table 2). Among the 13 patients with low TSH levels in the amiodarone group, there were 8 patients with mild subclinical hyperthyroidism (TSH 0.1$0.34 \mathrm{mU} / \mathrm{L}), 4$ patients with more significant subclinical hyperthyroidism (TSH $<0.1 \mathrm{mU} / \mathrm{L}$ ), and 1 patient with overt hyperthyroidism requiring immediate treatment with methimazole. 
There were 66 patients in the control group who were assigned to sotalol or placebo but treated with open-label amiodarone during follow-up. It was noted that 11 of these 66 patients developed subclinical hypothyroidism and 1 patient developed hyperthyroidism.

\section{Time to Elevated TSH}

Patients tended to develop hypothyroidism early in the study. Figure 2 displays the cumulative percentage of the TSH elevations that had been detected by month among the patients who developed hypothyroidism. Fifty-eight percent of patients who developed hypothyroidism in the amiodarone group and 50\% in the control group were detected at the first 3 months after treatment inception. Hypothyroidism was detected in approximately $76 \%$ of the patients in the amiodarone group and $80 \%$ in the control group by 6 months. Statistical differences in rates of elevated TSH levels were not found between the amiodarone and control groups. It also was noted that $91.7 \%$ of the patients on amiodarone who developed overt TSH elevations $(>10 \mathrm{mU} / \mathrm{L})$ had been detected at 6 months and $88.7 \%$ of the milder elevations in TSH levels $(4.5-10 \mathrm{mU} / \mathrm{L})$ had been detected at the end of the first year.

\section{Treatment with Levothyroxine}

Patients who developed elevated TSH levels while on amiodarone were more likely to have been treated with levothyroxine than similar patients in the control group. At 6 months and 1 year, $29.7 \%$ and $52.7 \%$, respectively, of the patients with elevated TSH levels in the amiodarone group were receiving supplemental levothyroxine. In contrast, none of the patients who developed hypothyroidism in the control group were treated with levothyroxine $(P<.0001)$. It also was noted that among the amiodarone group a significantly higher proportion of patients with overt hypothyroidism were treated with levothyroxine than those with subclinical hypothyroidism. At 6 months, $25.0 \%$ of the patients with overt hypothyroidism in the amiodarone group used levothyroxine, compared with $4.7 \%$ of the patients with subclinical hypothyroidism in the same group $(P=.002)$. Similarly, at 1 year, approximately $43 \%$ of patients in the amiodarone group with overt hypothyroidism were taking levothyroxine, whereas only $9.8 \%$ the patients with subclinical hypothyroidism were prescribed levothyroxine $(P<.0001)$.

\section{DISCUSSION}

Since amiodarone became available in the United States for anti-arrhythmic therapy in the 1980 s, there have been numerous reports of associated changes in thyroid hormone metabolism, as well as clinically significant thyroid dysfunction. ${ }^{10-15}$ Our study shows that amiodarone-induced hypothyroidism is common among a cohort of older men treated for atrial fibrillation as compared with a control group in a blinded study. If subclinical hypothyroidism, TSH $>4.5-10 \mathrm{mU} / \mathrm{L}$, is included, $31 \%$ of the patients on amioda- rone developed hypothyroidism. Although antibody testing was not done in our patients, the rate of positive antithyroid antibodies in this predominantly white male cohort is likely to be similar to the $12.9 \%-14.4 \%$ reported among a large population of 60-79-year-old white males in the NHANES III (National Health and Nutrition Examination Survey) study. ${ }^{16}$ Thus, despite a lower predisposition to thyroid dysfunction in men, nearly one third of all patients treated with amiodarone developed some degree of hypothyroidism, compared with $6.9 \%$ among the controls $(P<.0001)$. This incidence of high TSH levels among our control group is similar to the $8 \%-12 \%$ reported for men and women from 60-79 years of age in the NHANES III study. Because T4 levels were not consistently obtained during the follow-up period, we cannot determine the rate of overt hypothyroidism as defined by an elevated TSH level with low FT4. Instead, TSH levels above $10 \mathrm{mU} / \mathrm{L}$ were used as the cutoff for overt hypothyroidism, ${ }^{10}$ and were found among $5.0 \%$ of the amiodarone-treated group, compared with $0.3 \%$ in the control group $(P<.001)$.

Patients who developed elevations in their TSH levels did so early in both the amiodarone and control groups. At 6 months, $76 \%$ of the cases of hypothyroidism in the amiodarone group and $80 \%$ of the cases of hypothyroidism in the control group had been detected. Of the patients on amiodarone who developed TSH elevations above $10 \mathrm{mU} / \mathrm{L}, 92 \%$ had been detected at 6 months. A trend showed that hyperthyroidism, defined as TSH $<0.35 \mathrm{mU} / \mathrm{L}$, was more common among the amiodarone-treated patients. Because hyperthyroidism was a subclinical entity in all but 1 patient, and rare in absolute numbers, the analysis was directed toward only the hypothyroid patients.

Amiodarone contains 37\% iodine by weight. A 200-mg standard daily dose provides about 300 times the usual daily iodine intake. With initiation of treatment, the thyroid rapidly responds to the iodine load with a reduction in thyroid hormone synthesis, known as the Wolff-Chaikoff effect. Patients with normal thyroid function "escape" from the Wolff-Chaikoff effect by reducing thyroid iodide transport. ${ }^{12}$ Predictable changes of hormone levels that occur within the first week of therapy include a decrease in serum T3 levels and increase in serum reverse T3, T4, and TSH levels due to inhibition of type $15^{\prime}$-deiodinase in peripheral tissues and type $25^{\prime}$-deiodinase in the pituitary. ${ }^{13}$ After approximately 3 months of amiodarone, the compensatory increase in T4 levels reaches a steady state, and a majority of patients regain normal TSH levels. ${ }^{1}$ Through this escape mechanism, most patients remain clinically euthyroid while taking amiodarone. ${ }^{10}$ However, the large iodine load can lead to hypothyroidism in patients who do not escape from the Wolff-Chaikoff effect. Additionally, a more recent study by Tedelind et al reported that amiodarone and its iodidefree analog dronedarone are capable of inhibiting iodide uptake by thyroid follicular cells by way of an iodineindependent mechanism that does not involve the sodiumiodide symporter. ${ }^{14}$ 
Amiodarone-induced hypothyroidism is more common among populations with sufficient iodine intake, such as in the United States. One study comparing an iodine-sufficient area of Massachusetts and an iodine-deficient area of Italy found rates of amiodarone-induced hypothyroidism, as defined by elevated TSH levels and low or low-normal thyroxine level, to be $22 \%$ in Massachusetts and 5\% in Italy. ${ }^{15}$ Other risk factors for amiodarone-induced hypothyroidism include female sex and the presence of antithyroid antibodies. ${ }^{4,6}$ Antithyroid peroxidase antibodies are more common among white compared with black and Mexican-American individuals; $14.3 \%, 5.3 \%$, and $10.9 \%$, respectively. ${ }^{16}$ Among a group of 58 patients studied in the Netherlands with an overall incidence of amiodarone-induced hypothyroidism of $6.9 \%$, female sex and antithyroid antibodies were associated with relative risks of hypothyroidism of 7.9 and 7.3, respectively. Women with antithyroid antibodies had a combined relative risk for developing amiodarone-induced hypothyroidism of $13.5^{5}$

Less is known about the development of amiodaroneinduced hypothyroidism in men. Albert and colleagues reported the thyroid function tests of a male-predominant group on chronic amiodarone therapy followed for a mean of 27 months. ${ }^{17}$ Sixty-five percent of the 99 patients were male and all were euthyroid at baseline. Hypothyroidism was defined as a TSH level $>20$ with a thyroxine level less than the lower limit of normal. In the male subgroup, $30 \%$ became hypothyroid.

The laboratory diagnosis of hypothyroidism in patients on amiodarone therapy has evolved in the past 2 decades. Because of the absence of clinical features of hypothyroidism and the alteration of thyroid function tests by the blockade of T4 deiodination induced by amiodarone, ${ }^{18}$ resulting in elevation of serum $\mathrm{T} 4$ and free $\mathrm{T} 4$, the criterion for diagnosis of hypothyroidism was serum TSH $>14 \mathrm{mU} / \mathrm{L} .{ }^{19}$ However, in recent years there has been increasing interest in the effect of subclinical hypothyroidism on the cardiovascular system. ${ }^{20-23}$ Although there is debate about the benefits of treatment of patients with serum TSH levels of $4.5-10 \mathrm{mU} / \mathrm{L}$, there is general agreement that patients with subclinical hypothyroidism and serum TSH levels $>10$ $\mathrm{mU} / \mathrm{L}$ will benefit from treatment. ${ }^{24}$ It seems reasonable to apply this recommendation to patients on amiodarone, and in this study, $5.0 \%$ of patients on amiodarone warranted levothyroxine treatment.

It is evident from this study that practitioners are hesitant to start levothyroxine therapy in patients on amiodarone despite TSH levels $>10 \mathrm{mU} / \mathrm{L}$, possibly because of concern that the euthyroid state will cause recurrence of the atrial fibrillation. At 1 year, only $43 \%$ of patients with this degree of dysfunction on amiodarone were treated with levothyroxine. With this same level of hypothyroidism, an even lower proportion of patients on sotalol or placebo were treated with levothyroxine.

In regard to amiodarone-induced thyrotoxicosis, $5.3 \%$ of patients in this study developed low TSH levels while on amiodarone, as compared with $2.4 \%$ among the control group $(P=.07)$. Only 1 of the 13 patients with low TSH levels developed overt hyperthyroidism with clinical features of hyperthyroidism and elevated thyroxine level. The remaining 12 patients had subclinical hyperthyroidism that did not require antithyroid drugs or cessation of amiodarone. Recent prospective and controlled studies have implicated antithyroid antibodies ${ }^{4,6,25}$ and iodine deficiency in the pathogenesis. ${ }^{15}$ Our low rate of thyrotoxicosis is not completely unexpected in an iodine-sufficient area such as the United States. In one study done in iodine-sufficient Massachusetts, $2.0 \%$ of patients developed amiodarone-induced thyrotoxicosis compared with $10 \%$ in an iodinedeficient region of Italy. ${ }^{15}$ However, the reported prevalence of thyrotoxicosis has varied greatly depending on the group studied. Trip and colleagues followed 58 euthyroid patients, 72\% male, for a mean of 21 months in a moderately iodine-sufficient area of the Netherlands. ${ }^{5}$ The incidence of amiodarone-induced thyrotoxicosis was $12.1 \%$, and occurred at any time during therapy.

In summary, amiodarone-induced hypothyroidism developed in $30.8 \%$ of older men treated with amiodarone for chronic atrial fibrillation compared with the control group and presented early during therapy. Serum TSH levels $>10$ $\mathrm{mU} / \mathrm{L}$ were found in $5.0 \%$ of amiodarone-treated men, and this should warrant treatment with thyroxine. Hyperthyroidism occurred in 5.3\% of amiodarone-treated patients, but was a subclinical entity in all but 1 case and not statistically different from the rate in the control group. Given the high rate of hypothyroidism among patients taking amiodarone, monitoring of thyroid function is recommended at baseline, 3 months, and every 6 months thereafter during the therapy.

\section{References}

1. Melmed S, Nademanee K, Reed AW, et al. Hyperthyroxinemia with bradycardia and normal thyrotropin secretion after amiodarone administration. J Clin Endocrinol Metab. 1981;53:997-1001.

2. Martino E, Bartalena L, Bogazzi F, Braverman LE. Effects of amiodarone on the thyroid. Endocr Rev. 2001;22(2):240-254.

3. Harjai KJ, Licata AA. Effects of amiodarone on thyroid function. Ann Intern Med. 1997;126:63-73.

4. Martino E, Aghini-Lombardi F, Mariotti S, et al. Amiodarone iodineinduced hypothyroidism: risk factors and follow-up in 28 cases. Clin Endocrinol. 1987;26:227-237.

5. Trip MD, Wiersinga W, Plomp TA. Incidence, predictability and pathogenesis of amiodarone induced thyrotoxicosis and hypothyroidism. Am J Med. 1991;91(5):507-511.

6. Martino E, Aghini-Lombardi F, Bartalena L, et al. Enhanced susceptibility to amiodarone-induced hypothyroidism in patients with anutoimmune thyroid disease. Arch Intern Med. 1994;23:2722-2726.

7. Singh BN, Singh SN, Reda DJ, et al. Amiodarone Atrial Fibrillation Efficacy Trial (SAFE-T) Investigators. Amiodarone versus sotalol for atrial fibrillation. Veterans Affairs Cooperative Study. $N$ Engl J Med. 2005;352:1861-1872.

8. Wahlberg P, Wennstrom J, Ekelund P. Control of thyrotoxicosis with sotalol. Ann Clin Res. 1976;8:415-417.

9. Perrild H, Hansen JM, Skovsted L, Christensen LK. Different effects of propranolol, alprenolol, sotalol, atenolol and metoprolol on serum T3 and serum rT3 in hyperthyroidism. Clin Endocrinol. 1983; 18:139-142. 
10. Nademanee K, Singh BN, Callahan B, et al. Amiodarone, thyroid hormone indexes and altered thyroid function: long term serial effects in patients with cardiac arrhythmias. Am J Cardiol. 1986;58:981-986.

11. Kennedy RL, Griffiths H, Gray TA. Amiodarone and the thyroid. Clin Chem. 1989;35:1882-1887.

12. Wiersinga WM, Trip MD. Amiodarone and thyroid hormone metabolism. Postgrad Med J. 1986;62:909-914.

13. Franklyn JA, Gammage MD, Sheppard MC. Amiodarone and thyroid hormone effects on anterior pituitary hormone gene expression. Clin Endocrinol (Oxf). 1987;27:373-382.

14. Tedelind S, Larsson F, Johanson C, et al. Amiodarone inhibits thyroidal iodine transport in vitro by a cyclic AMP and iodine independent mechanism. Endocrinology. 2006;147:2936-2943 (Epub 2006 Mar 9).

15. Martino E, Safran M, Aghini-Lombardi F, et al. Environmental iodine intake and thyroid dysfunction during chronic amiodarone therapy. Ann Intern Med. 1984;101:28-34.

16. Hollowell JG, Staehling NW, Flanders D, et al. Serum TSH, T4, and thyroid antibodies in the United States population (1988 to 1994): National Health and Nutrition Examination Survey (NHANES III). J Clin Endocrinol Metab. 2002;87:489-499.

17. Albert SG, Alves LE, Rose EP. Thyroid dysfunction during chronic amiodarone therapy. J Am Coll Cardiol. 1987;9:175-183.
18. Hershman JM, Nademanee K, Sugawara M, et al. Thyroxine and triiodothyronine kinetics in cardiac patients taking amiodarone. Acta Endocrinol. 1986;111:193-199.

19. Nademanee K, Piwonka P, Singh BN, Hershman JM. Amiodarone and thyroid function. Prog Cardiovasc Dis. 1989;31:427-437.

20. Biondi B, Klein I. Hypothyroidism as a risk factor for cardiovascular disease. Endocrine. 2004;24:1-13.

21. Rondondi N, Newman AB, Vittinghoff E, et al. Subclinical hypothyroidism and the risk for heart failure, other cardiovascular events and death. Arch Intern Med. 2005;165:2460-2466.

22. Walsh JP, Bremner AP, Bulsara MK, et al. Subclinical thyroid disease as a risk factor for cardiovascular disease. Arch Intern Med. 2005;165: 2467-2472.

23. Cappola A, Fried L, Arnold A, et al. Thyroid status, cardiovascular risk and mortality in older adults. JAMA. 2006;295:1033-1041.

24. Surks MI, Ortiz E, Daniels GH, et al. Subclinical thyroid disease: scientific review and guidelines for diagnosis and management. JAMA. 2004;291:228-238.

25. Martino E, Macchia E, Aghini-Lombardi F, et al. Is humoral thyroid autoimmunity relevant in amiodarone iodine-induced thyrotoxicosis AIIT? Clin Endocrinol. 1986;24:627-633. 